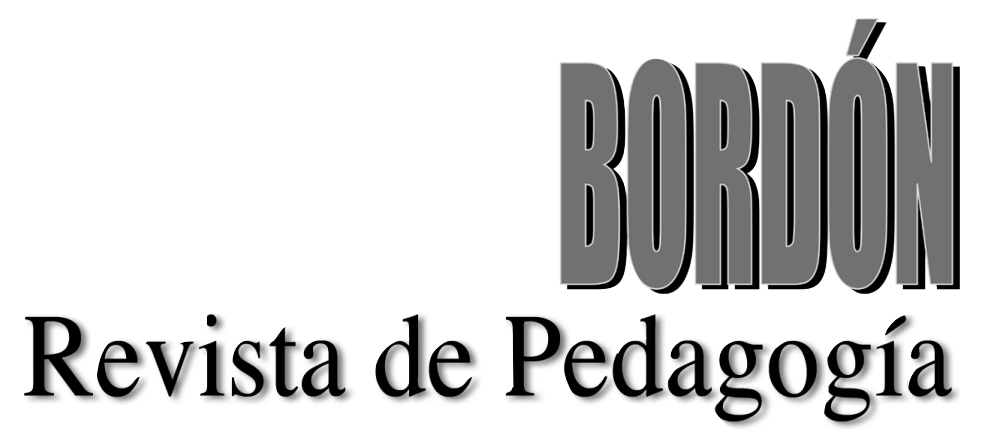

NÚMERO MONOGRÁFICO / SPECIAL ISSUE

Formación inicial de maestros / Pre-primary and primary teacher training and education

Miquel Martínez

(editor invitado / guest editor)

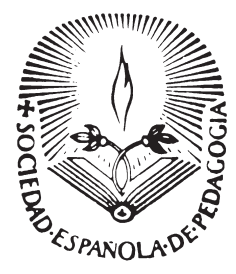

Volumen 68

Número, 2

2016 


\title{
RELACIÓn ERTRE LAS PREFERENCIAS DE FORMACIÓn DEL PROFESORADO Y SU COMPETENCIR DIGITAL EN LAS REDES SOCIALES
}

\section{The relationship between teacher training preferences and their digital skills on social networks}

\author{
RAFAEL GARCÍA-PÉREZ('),ÁNGELES REBOLLO-CATALÁN ${ }^{(1)}$ Y CURRO GARCÍA-PÉREZ(2) \\ (1) Universidad de Sevilla \\ (2) CEP Algeciras-La Línea
}

D01: 10.13042/Bordon.2016.68209

Fecha de recepción: 20/09/2015 - Fecha de aceptación: 09/02/2016

Autor de contacto / Corresponding Author: Rafael García-Pérez. Email: rafaelgarcia @us.es

INTRODUCCIÓN. Diversos informes internacionales han revelado la importancia de la formación del profesorado en TIC como un factor clave para la innovación y la mejora educativa. Estudios recientes analizan las competencias del profesorado para aprovechar el potencial pedagógico de las tecnologías digitales. Con este artículo pretendemos estudiar la relación entre las preferencias de formación del profesorado y su competencia digital en el uso de las redes sociales, lo que nos permite descubrir cuáles son las modalidades de formación más efectivas para lograr un nivel avanzado de competencia digital. MÉTODO. Se ha llevado a cabo una investigación basada en encuesta a una muestra de 1.701 profesores procedentes de 114 centros educativos de la zona CEP Algeciras-La Línea, Cádiz (Andalucía, España). Se aplican sendas escalas de competencias digitales y preferencias de aprendizaje diseñadas ad hoc con coeficientes de fiabilidad de .92 y .82 respectivamente. RESULTADOS. El profesorado muestra un nivel moderado de competencias digitales siendo las competencias docentes las menos desarrolladas. El profesorado con una alta predisposición a las diversas modalidades de formación junto con el que muestra preferencias hacia modalidades colaborativas en combinación con el aprendizaje autónomo son los que expresan un nivel más avanzado de competencia digital. No se observan diferencias en la competencia digital del profesorado en función del género pero sí en función de la edad. DISCUSIÓN. Estos resultados concuerdan con los de otras investigaciones sobre formación del profesorado en TIC, que muestran deficiencias en la formación del profesorado para un mejor aprovechamiento pedagógico de las TIC. Se aportan evidencias que permiten extraer conclusiones para orientar la formación del profesorado en TIC.

Palabras clave: Competencias del profesorado, Usos de la tecnología en educación, Redes sociales, tecnologías de la web 2.0, Formación del profesorado, Competencia digital. 


\section{Introducción}

La educación en la actualidad se enfrenta a importantes desafíos derivados de la irrupción de las tecnologías de la información y la comunicación y la llegada de la sociedad del conocimiento. Informes internacionales (Comisión Europea, 2015; Ferrari, 2013; OCDE, 2014) han revelado la importancia de la formación del profesorado en TIC como un factor clave para la innovación y la mejora educativa. Según el INTEF (2013), aunque España es el país de la Unión Europea que mayor número de horas de formación en TIC dedica por docente, el profesorado considera baja su capacitación para una plena integración de los medios tecnológicos, poniendo de relieve que pocos profesores crean recursos digitales o participan en la página web de su centro, en entornos virtuales de aprendizaje o en medios sociales de colaboración profesional. Por ello, tomando como referencia el informe DIGCOMP (Ferrari, 2013), el INTEF (2013) propone el Plan de Cultura Digital en la Escuela, incluyendo la competencia digital docente como una línea prioritaria para la formación del profesorado.

Paralelamente, la Web 2.0 con sus nuevos recursos para la comunicación y la colaboración está introduciendo cambios en la naturaleza y relación entre educación formal e informal tendiendo puentes y acortando la distancia entre ambas (Castañeda y Adell, 2013; Coll y Engel, 2014; Greenhow y Robelia, 2009; Tyner, Gutiérrez-Martín y Torrego-González, 2015).No obstante, como ha señalado Fahser-Herro y Steinkuehler (2010), aunque las tecnologías de la web 2.0 están introduciendo cambios en la forma de concebir la educación al plantear formas colaborativas y no lineales de construir y compartir conocimiento, la inclusión de esta nueva visión en los programas y cursos de formación inicial y continua del profesorado todavía llevará tiempo.

En un estudio reciente, Cortina-Pérez, GallardoVigil, Jiménez-Jiménez y Trujillo-Torres (2014) encuentran que más del $60 \%$ del profesorado reconoce no hacer ningún uso de las herramientas 2.0 en su actividad docente, constatando también que el profesorado no se considera suficientemente formado en ellas para hacer frente a su tarea docente. Aunque más del 90\% ha recibido formación relacionada con las TIC, los datos obtenidos detectan una importante carencia formativa, con más del 70\% de los encuestados que aseguran no sentirse preparados para hacer un uso educativo de las herramientas 2.0. También San Nicolás, Fariñas y Area (2012) encuentran que el profesorado hace un escaso uso de espacios de la Web 2.0, como las redes sociales, foros de debate y blogs, aunque cuenta con un adecuado nivel y dominio de las TIC. En su estudio transversal, Hughes, Ko, Lim, y Liu (2015) demuestran que casi todo el profesorado en formación utilizaba Facebook pero lo hacía solo para uso personal, encontrando que el uso de las redes sociales para actividades profesionales aumentó de un $7 \%$ a un $22 \%$ de 2008 a 2012. Almerich, Suárez-Rodríguez, Jornet y Orellana (2011a) también encontraron más competencias en el profesorado a nivel personal-profesional que docente de aula, demostrando, a su vez, la relación entre la escasa confianza del profesorado y su bajo nivel de competencias. En su estudio sobre el uso de las TIC en educación a nivel europeo, Wastiau et al. (2013) afirman que hay una relación entre la competencia digital del profesorado y el uso de las TIC en el aula, encontrando que aquellos que tienen más confianza en sus habilidades digitales y en el impacto positivo de las TIC en el aprendizaje, desarrollan más actividades basadas en TIC con sus estudiantes, lo que tiene un efecto positivo en la adquisición de habilidades digitales del alumnado y en su confianza al usar estas herramientas. Suárez-Rodríguez, Almerich, Gargallo y Aliaga (2013) también encontraron que las competencias tecnológicas junto con la percepción y creencias de autoeficacia influían en las competencias pedagógicas del profesorado en el uso de las TIC. Más recientemente, Wang, Xu y Chuan (2015) también prueban la influencia de la autoeficacia percibida con el ordenador en la experiencia de uso de Facebook. La investigación internacional 
también ha mostrado suficientes evidencias sobre la brecha digital por factores sociales como la edad, la clase social, el género, el nivel educativo o la ubicación geográfica (DiMaggio, Hargittai, Celeste y Shafer, 2004; Livingstone y Helper, 2007; Hargittai y Hinnant, 2008). No obstante, los datos parecen indicar que la brecha digital de género se reduce en el uso de las redes sociales virtuales (Clipson, Wilson y DuFrene, 2012; Mazman y Usluel, 2011). Los estudios sobre competencias digitales del profesorado también muestran esta tendencia, encontrando diferencias en función del género y la edad a favor de los hombres y los más jóvenes en el uso de tecnologías en general, excepto en el caso del uso de las redes sociales (Almerich et al., 2011b; CortinaPérez et al., 2014; Suárez et al., 2013).

Algunas investigaciones previas (Rebollo-Catalán, Vico-Bosch y García-Pérez, 2015; Vergés, Hache y Cruel, 2011) han mostrado una relación entre la heterogeneidad de modalidades de formación y el logro de competencias tecnológicas avanzadas. En el proyecto "El futuro digital de la formación del profesorado", desarrollado en el marco del programa Recursos Educativos Abiertos del Reino Unido, Gruszczynska, Merchant y Pountney (2013) encuentran que las prácticas digitales más interesantes en la escuela han surgido de la labor de profesores innovadores entusiastas, trabajando a menudo solos o en colaboración con investigadores, o como parte de redes informales de base. Wastiau et al. (2013) han mostrado que el profesorado prefiere métodos informales de formación sobre el uso de las TIC, estrategias de formación semipresencial y una formación basada en situaciones reales de aula.

Almerich, Suárez-Rodríguez, Belloch y Bo (2011b) señalan tres aspectos que han condicionado la eficacia de los programas de formación del profesorado en TIC: 1) la focalización en aspectos tecnológicos más que pedagógicos; 2) el desajuste de la formación a los conocimientos y habilidades del profesorado y, 3) la falta de tiempo para llevar a cabo innovación con TIC junto con un insuficiente seguimiento de su aplicación en el aula. Según estos autores, el incremento de la eficacia y calidad de estos programas reside no solo en un desarrollo apropiado de los conocimientos y habilidades en TIC sino también y muy especialmente en la adecuación de estos a las necesidades del profesorado. Estos autores argumentan que los programas de desarrollo profesional del profesorado se han basado con frecuencia en estándares nacionales o en la opinión de expertos en formación y, raramente, en la opinión del profesorado, lo que ha podido incidir en la falta de ajuste entre la formación ofrecida y las necesidades del profesorado. Cabero (2014) va más allá al plantear que es la propia formación la que debe cambiar para dar más valor a la práctica y la reflexión sobre la misma, contemplando problemas reales para los docentes, no para los formadores o los técnicos, por lo que el diseño y planificación de la misma debería contar con la participación activa del profesorado para que se ajuste a sus necesidades.

Sadaf, Newby y Ertmer (2013) demostraron que aunque el profesorado en formación mostraba una fuerte intención a usar wikis, blogs y redes sociales en sus futuras clases para mejorar el aprendizaje del alumnado, la interacción entre estudiante-estudiante y estudiante-profesorado, el aprendizaje colaborativo, las habilidades de escritura del alumnado y para compartir conocimiento de la materia, este consideraba que un uso exitoso dependería de una integración significativa de la Web 2.0 con los contenidos de la materia, con las metas de aprendizaje y con el nivel de edad del alumnado. Ugur (2014) demuestra que, aunque los profesores en formación tenían capacidad para planificar su enseñanza incluyendo herramientas de la Web 2.0, su práctica dependía más del contexto institucional (programas, organización, etc.) y del contenido curricular, concluyendo que la formación en TIC del profesorado debería contemplar estrategias basadas en contenidos específicos y en actividades de modelado para una efectiva integración de las TIC en las prácticas educativas en el aula. Keith (2013) señala que 
incluso el profesorado más entusiasta se frena en el uso de estas tecnologías por las políticas institucionales de cortafuegos que bloquean el acceso y uso de las redes sociales virtuales en los contextos escolares por lo que recomienda que los programas de formación del profesorado incluyan no solo las ventajas y beneficios que reportan las redes sociales virtuales para el aprendizaje sino también los riesgos potenciales con el fin de garantizar la seguridad, privacidad y bienestar psicológico de los estudiantes al tomar las precauciones adecuadas.

Aunque las redes sociales han irrumpido con gran fuerza en las prácticas y hábitos sociales, con mayor incidencia en las generaciones jóvenes y son las principales responsables del aumento en el uso de las tecnologías móviles, pocos estudios se centran en las competencias del profesorado en el uso de las mismas con especial hincapié en usos docentes y, menos aún, indagan en qué estrategias de formación son más efectivas en el logro de competencias digitales avanzadas.

\section{Planteamiento y objetivos del estudio}

Aunque como hemos comentado con anterioridad, la competencia digital docente es una problemática actual que ha originado investigaciones a nivel internacional, la mayor parte de las publicaciones recientes se centran en el profesorado en formación y pocas en el profesorado en activo. Igualmente la mayor parte de las investigaciones recientes publicadas exploran las competencias tecnológicas del profesorado y, en menor medida, las competencias digitales, siendo muy pocos los estudios que analizan específicamente los usos docentes de las redes sociales virtuales. Las investigaciones que se han publicado sobre profesorado y tecnologías de la Web 2.0 de los últimos cinco años se centran en su uso para el desarrollo profesional docente, siendo las wikis, blogs y foros las herramientas que predominan $y$, en menor medida, las redes sociales.
Creemos de interés en un momento de cambio y revisión de los programas de formación continua del profesorado, estudiar cuáles son los perfiles formativos de profesorado que alcanzan un nivel avanzado de competencia digital en el uso de las redes sociales. Nuestra hipótesis de partida es que el profesorado que usa de forma combinada una variedad de estrategias formativas es el que alcanza un mayor nivel de competencia digital en las redes sociales, siendo las fórmulas colaborativas y autodidactas las más efectivas para el logro de competencias digitales avanzadas en el uso de las redes sociales.

Por todo ello, los objetivos específicos que nos planteamos con nuestro trabajo son:

1) Describir el nivel de competencia digital del profesorado en el uso de las redes sociales virtuales.

2) Identificar perfiles de profesorado según sus preferencias de formación en TIC.

3) Caracterizar dichos perfiles según su nivel de competencia digital en las redes sociales.

\section{Metodología}

\section{Diseño de investigación}

Esta investigación se puede englobar en lo que se denominan metodologías ex post facto, adoptando un diseño correlacional a través de estudios de cuestionario de auto-informe, con medidas de comparación de grupos en función de edad y género. Con este diseño no pretendemos modificar las variables objeto de estudio sino explorar su naturaleza y comportamiento en la muestra analizada y estudiar las relaciones que se expresan entre ellas.

\section{Participantes}

La muestra del estudio se compone de 1.701 docentes de la zona CEP Algeciras-La Línea 
(Cádiz, España) procedentes de 114 centros públicos. Este tamaño permite trabajar con un error inferior al $\pm 2 \%$ para un nivel de confianza del $95,5 \%$ y p=q. La muestra invitada es el total de la población de profesorado en activo durante el curso 2014-2015, la cual asciende a 3.500 profesores procedentes de 120 centros. La muestra final participante supone el $49 \%$ de esta población.

Del profesorado encuestado, el 31,7\% son hombres y el 68,3\% mujeres. Por etapa educativa, el $43 \%$ son de educación primaria, el $31,1 \%$ de enseñanza secundaria, el $17,1 \%$ de educación infantil, el 6,9\% de ciclos formativos y el 1,8\% de régimen especial. La tabla 1 resume las características de la muestra del profesorado participante.

\section{Instrumentos}

El cuestionario empleado incluye las siguientes secciones:

a) Características sociodemográficas y de uso de las tecnologías: se pregunta a los participantes por su género, edad, antigüedad docente, etapa educativa, situación laboral, cargos académicos recientes, formación recibida y participación en planes y programas, con el propósito de obtener una descripción de la muestra y estudiar su posible influencia sobre otras variables del estudio.

b) Escala de competencias digitales en redes sociales: elaborada ad hoc a partir de estudios previos (Area y Pessoa, 2012; Janssen

TABLA 1. Datos del profesorado participante

Variables

\begin{tabular}{|c|c|c|c|}
\hline Sexo & $\begin{array}{l}\text { Mujer } \\
\text { Hombre }\end{array}$ & $\begin{array}{r}1.134 \\
527\end{array}$ & $\begin{array}{l}68,3 \% \\
31,7 \%\end{array}$ \\
\hline Edad & $\begin{array}{l}\leq 30 \text { años } \\
31-40 \text { años } \\
41-50 \text { años } \\
\geq 51 \text { años }\end{array}$ & $\begin{array}{l}152 \\
602 \\
505 \\
414\end{array}$ & $\begin{array}{r}9,1 \% \\
36,0 \% \\
30,2 \% \\
24,7 \%\end{array}$ \\
\hline Situación laboral & $\begin{array}{l}\text { Funcionario } \\
\text { Interino }\end{array}$ & $\begin{array}{r}1.192 \\
441\end{array}$ & $\begin{array}{l}73,0 \% \\
27,0 \%\end{array}$ \\
\hline Antigüedad docente & $\begin{array}{l}\leq 5 \text { años } \\
6-10 \text { años } \\
11-15 \text { años } \\
16-20 \text { años } \\
21-25 \text { años } \\
\geq 26 \text { años }\end{array}$ & $\begin{array}{l}372 \\
342 \\
259 \\
182 \\
208 \\
289\end{array}$ & $\begin{array}{l}22,5 \% \\
20,7 \% \\
15,7 \% \\
11,0 \% \\
12,6 \% \\
17,5 \%\end{array}$ \\
\hline Etapa educativa & $\begin{array}{l}\text { Infantil } \\
\text { Primaria } \\
\text { Secundaria } \\
\text { Ciclo formativo } \\
\text { Régimen especial }\end{array}$ & $\begin{array}{r}273 \\
683 \\
493 \\
109 \\
29\end{array}$ & $\begin{array}{r}17,2 \% \\
43,0 \% \\
31,1 \% \\
6,9 \% \\
1,8 \%\end{array}$ \\
\hline $\begin{array}{l}\text { Cargos académicos recientes } \\
\text { (no excluyentes) }\end{array}$ & $\begin{array}{l}\text { Tutor/a } \\
\text { Jefe/a departamento } \\
\text { Coordinador de ciclo } \\
\text { Equipo directivo }\end{array}$ & $\begin{array}{l}983 \\
234 \\
199 \\
213\end{array}$ & $\begin{array}{l}57,8 \% \\
13,8 \% \\
11,7 \% \\
12,5 \%\end{array}$ \\
\hline
\end{tabular}

Fuente: elaboración propia. 
et al., 2013), la cual consta de 30 ítems agrupados en seis categorías que miden competencias instrumentales (habilidades de uso de las redes con fines elementales - terminología, navegación, funcionalidad-), cognitivas (habilidades para reunir, organizar, analizar y juzgar la relevancia y propósito de la información digital), comunicativas (habilidades para conectar, comunicar y colaborar con otras personas a través de las redes), éticas (habilidades para usar las redes de forma responsable, segura y sostenible), emocionales (habilidades para mantener una actitud abierta, curiosa y equilibrada en el uso de las redes y para expresar, comprender y regular emociones) y docentes (habilidades para aplicar las redes con fines educativos) y cuya respuesta es de tipo Likert de cuatro puntos (de 0 -nunca- a 3 -siempre-). Tras aplicar un análisis de componentes principales categórico con un procedimiento de escalamiento óptimo para datos ordinales, obtenemos un coeficiente alpha de Cronbach de .92 e índice altos de saturación de todos los ítems en el componente principal con una media de .534 y una desviación típica de .122 que muestran la unidimensionalidad de la escala, lo que nos indica una alta fiabilidad y una óptima validez de constructo. Para reducir el sesgo derivado por las medidas de autoinforme, hemos aplicado, de forma complementaria, la escala de autoeficacia percibida en el uso de TIC de Howard (2014) como medida de validez concurrente (Rho $=.434 ; \mathrm{p} \leq .01$ ). De forma complementaria, se calcula la fiabilidad de las subdimensiones de la escala (tecnológica $=.83$; cognitiva $=.80$; socio-comunicativa $=.80$; ética $=.77$; emocional $=.69$; docente $=.87$ ), configurando cada una de ellas una variable cuya medida oscila de 0 a 15 puntos.

c) Escala de preferencias sobre las modalidades de formación en TIC: consta de 10 ítems agrupados en tres categorías que miden las preferencias en modalidades de formación convencional, colaborativa y autónoma cuya respuesta es de tipo Likert de cuatro puntos (de 0 -nunca- a 3 siempre-). El escalamiento óptimo mediante análisis de componentes principales categórico arroja un coeficiente alpha de Cronbach de .82, mostrando que se trata de una medida fiable, así como de índices altos de saturación de todos los ítems en el componente principal ( $\mathrm{M}=$ .612 ; DT $=.056$ ), lo que revela una medida válida de esta variable.

\section{Procedimiento}

Para la recogida de datos, se contactó con los equipos directivos de los centros a los que se informó en reuniones personales del propósito de la investigación y se les propuso la participación en esta investigación mediante la cumplimentación de una encuesta sobre competencia digital y uso de las redes sociales de una duración de 30 minutos por parte del profesorado adscrito a su centro, acordando la entrega de un informe global a cada centro con un nivel de participación de su profesorado de al menos el 50\%. Se entrega a los centros un protocolo de consentimiento informado para garantizar que todo el profesorado es debidamente informado del carácter voluntario y anónimo de su participación en el estudio y de los objetivos del mismo. El profesorado aceptó participar voluntariamente sin recibir ninguna compensación a cambio mediante la firma de este protocolo que nos envía previamente a la realización de la encuesta. Una vez recibidas las encuestas en los centros se les propone el plazo de un mes para la realización y entrega. Finalmente, la recogida de datos se realiza durante los meses de abril y mayo de 2015.

Los datos fueron introducidos en el paquete estadístico SPSS (versión 20). Una vez comprobada la unidimensionalidad de las escalas competencias digitales y de preferencias de formación con el análisis de componentes principales, 
creamos una variable de cada una de ellas con la puntuación global a partir del sumatorio de todos los ítems, quedando la variable global de competencia digital en una escala de 0 a 90 puntos y la variable global de preferencias de formación en una escala de 0 a 30 puntos. Para este trabajo, presentamos los resultados de la competencia digital global en una escala de tres puntos (0-29 = Básica; 30-59 = moderada; y, 6090 =avanzada), una vez comprobada que dicha recodificación no afecta a su validez y fiabilidad y que se ajusta a los estándares internacionales de medida de las habilidades digitales.

También aplicamos un análisis factorial de componente principal con rotación varimax a la medida de las preferencias de formación que arroja una solución en tres factores que explica el $66,35 \%$ de la varianza total $(23,77 \%$ el factor $1 ; 21,92 \%$ el factor $2 ; 20,66 \%$ el factor 3$)$. El análisis de la fiabilidad indica un alpha de Cronbach .74 para el factor 1 (convencional), .72 para el factor 2 (autónoma) y .74 para el factor 3 (colaborativa). Todos los ítems obtuvieron pesos factoriales superiores a $.50 \mathrm{en} \mathrm{el}$ factor al que fueron asignados (tabla 2), tomando como criterio asignar el ítem al factor donde su peso fuera mayor.

Para el tratamiento estadístico de los datos, hemos realizado un análisis exploratorio de las variables en estudio para conocer las características de su distribución. Posteriormente, realizamos un análisis de conglomerados de $\mathrm{K}$ medias (Q-Cluster) para establecer perfiles de profesorado en función de sus valores en las subdimensiones de la escala de preferencias de formación en TIC y un análisis discriminante para caracterizar los perfiles en función de su nivel de competencia digital. Por último, una

Tabla 2. Matriz de componentes rotados

Ítems

Factor $1 \quad$ Factor $2 \quad$ Factor 3

Convencional Autónoma Colaborativa

Ítem 5. Asistiendo a cursos sobre herramientas y aplicaciones

, 798

específicas y útiles

Ítem 4. Realizando cursos virtuales que me permiten ir aplicando $\quad$, 363

lo que aprendo

Ítem 8. Asistiendo a cursos sobre cómo funcionan a nivel básico para $\quad, 706$

después...

Ítem 3. Usándola de forma intuitiva por el placer de experimentar

con la tecnología

Ítem 2. Indagando y probando mientras intento hacer cosas que me gustan

\begin{tabular}{|c|c|c|c|}
\hline Ítem 9. Dialogando con otras personas en foros y grupos & ,305 &, 540 &, 377 \\
\hline Ítem 1. Participando con otras personas en proyectos comunes & & & ,848 \\
\hline $\begin{array}{l}\text { Ítem } 6 \text {. Formando parte de un grupo o comunidad que me haga sentir } \\
\text { su apoyo }\end{array}$ & ,330 & &, 764 \\
\hline $\begin{array}{l}\text { Ítem 10. Apuntándome a actividades organizadas por asociaciones } \\
\text { y grupos que conozco }\end{array}$ &, 515 & &, 532 \\
\hline
\end{tabular}

Método de extracción: análisis de componentes principales

Método de rotación: normalización varimaxKaiser

La rotación ha convergido en 5 iteraciones

Fuente: Elaboración propia. 
vez aplicada la prueba Kolmogorov Smirnov con la que se rechaza la hipótesis de normalidad de las distribuciones, se aplican las pruebas de contraste no paramétricas chi cuadrado, U de Mann-Whitney y Anova de Kruskal-Wallis para muestras independientes con el objeto de estudiar si existen diferencias estadísticamente significativas en el nivel de competencia digital del profesorado en función de género edad perfiles formativos de profesorado. Aplicamos la d de Cohen para valorar la magnitud de las diferencias.

\section{Resultados}

\section{Competencias digitales del profesorado}

Los resultados indican un nivel moderado de competencia digital $(\mathrm{M}=47,9 ; \mathrm{DT}=17,85)$, encontrando que un $13 \%$ del profesorado muestra un nivel básico de competencias, un 62,3\% expresa un nivel moderado y el $24,7 \%$ presenta un nivel avanzado de competencias digitales en el uso de las redes sociales. No se observan diferencias en el nivel global de competencia digital del profesorado función del género $\left(X^{2}=6,237\right.$; $\mathrm{p}=.044 ; \mathrm{C}$.Contingencia $=.066)$ pero sí en función de la edad $\left(\mathrm{X}^{2}=62,786 ; \mathrm{p}=.000 ; \mathrm{C}\right.$ .Contingencia $=.204$ ), siendo el grupo de profesorado menor de 41 años el que presenta mayor nivel de competencia digital.

El profesorado obtiene puntuaciones por encima de la mediana en 15 competencias (figura 1), alcanzando puntuaciones cercanas al valor máximo de la escala en cinco de ellas: capacidad para usar las redes sin perjuicio de otras actividades ( $M=2,35$; DT $=0,919)$, no molestar y actuar con respeto $(\mathrm{M}=2,32 ; \mathrm{DT}=0,971)$, mostrar alegría empática $(\mathrm{M}=2,31 ; \mathrm{DT}=0,908)$, expresarse con claridad( $\mathrm{M}=2,13$; $\mathrm{DT}=0,875)$, y manejarlas a través de dispositivos móviles $(\mathrm{M}=2,15 ; \mathrm{DT}=0,954)$. Más del $80 \%$ del profesorado maneja estas competencias con fluidez, lo que expresa competencia en la creación de climas relacionales saludables en las redes.

FIGURA 1. Ranking de competencias digitales del profesorado en redes sociales

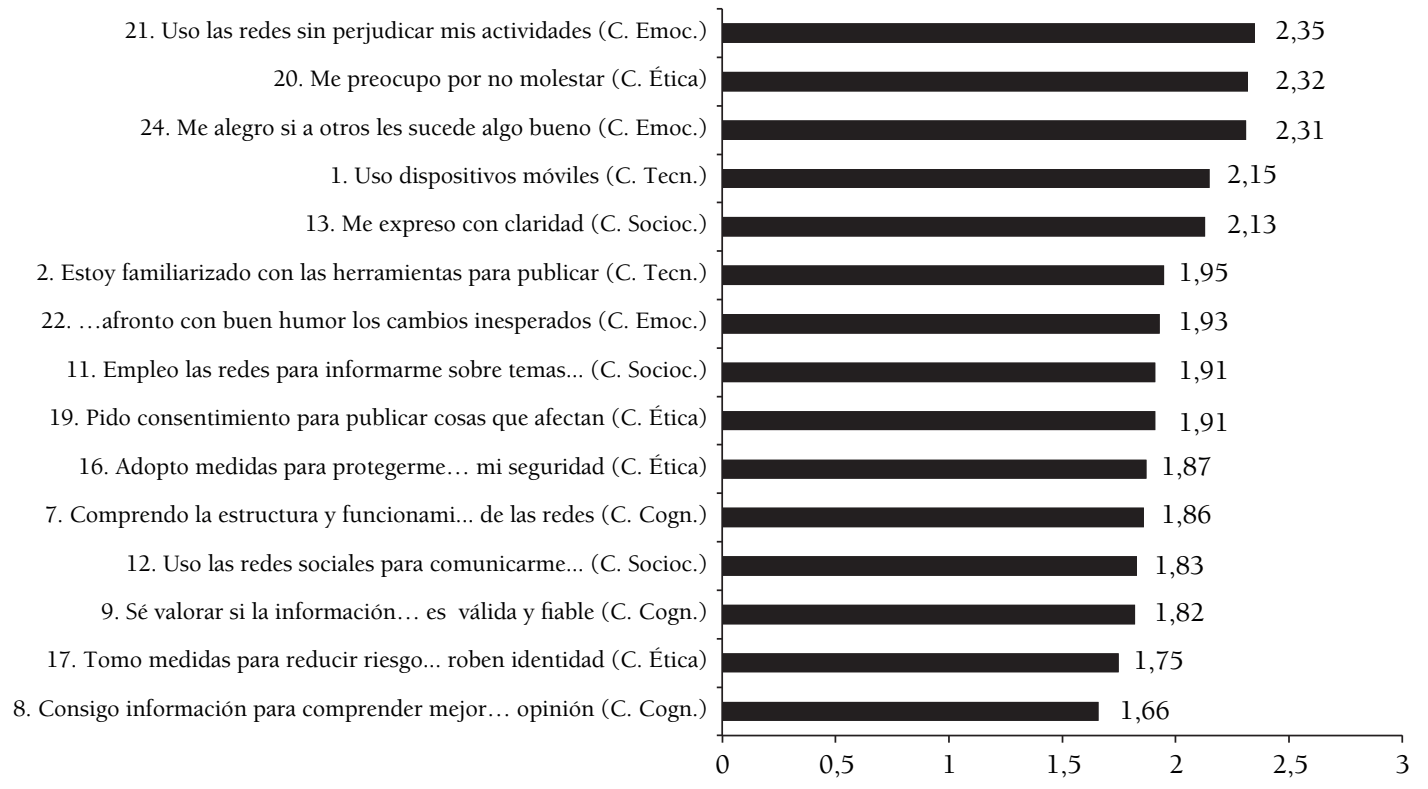


Figura 2. Medias del profesorado en las dimensiones de la competencia digital

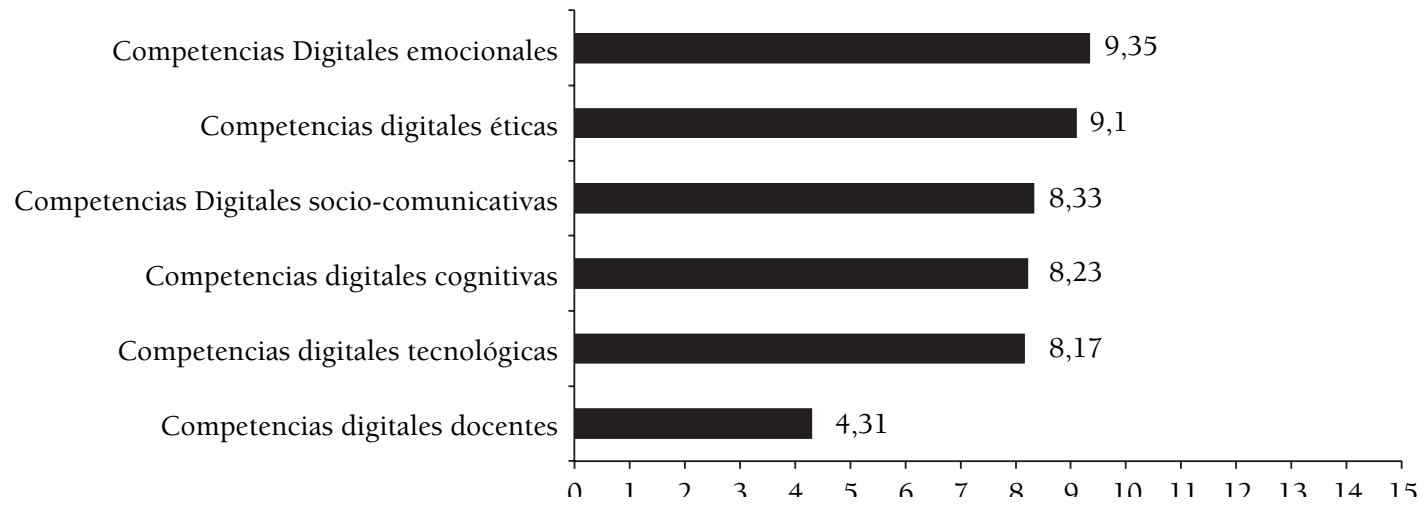

En contraste, el profesorado obtiene puntuaciones muy bajas en capacidad para trabajar temas académicos con su alumnado $(\mathrm{M}=0,98 ; \mathrm{DT}=$ 0,907), para usarlas como medio didáctico $(\mathrm{M}=0,79$; $\mathrm{DT}=0,941)$, para ampliar la comunidad educativa virtual $(\mathrm{M}=0,70 ; \mathrm{DT}=0,875)$ y para implicar al alumnado en proyectos colaborativos $(\mathrm{M}=0,63 ; \mathrm{DT}=0,797)$. Esto muestra que el profesorado obtiene una baja puntuación en competencias digitales de uso docente de las redes sociales ( $M=4,31 ; \mathrm{DT}=3,705)$ (figura 2). Solo el $11,2 \%$ del profesorado alcanza un nivel óptimo en estas competencias.

Los resultados muestran diferencias significativas en competencia digital del profesorado en función del uso docente de las redes $\left(\mathrm{X}^{2}=\right.$ 376,282; p=.000; C.Contingencia $=.454$ ), encontrando que es el profesorado con un nivel avanzado de competencia digital el que más usa las redes con fines docentes con su alumnado, mientras que el profesorado con niveles moderado o básico de competencia digital lo hace muy poco (tabla 3 ).

Perfiles del profesorado según sus preferencias de formación en TIC

Los resultados revelan una correlación significativa entre las preferencias de formación y la competencia digital (Rho $=.396^{* *}$ ), indicando que a más diversidad en preferencias formativas más competencias digitales. El análisis de conglomerados de las preferencias de formación en TIC nos permite identificar seis perfiles de profesorado bien diferenciados (tabla 4).

TABla 3. Tabla de contingencia competencia digital global y competencia digital docente

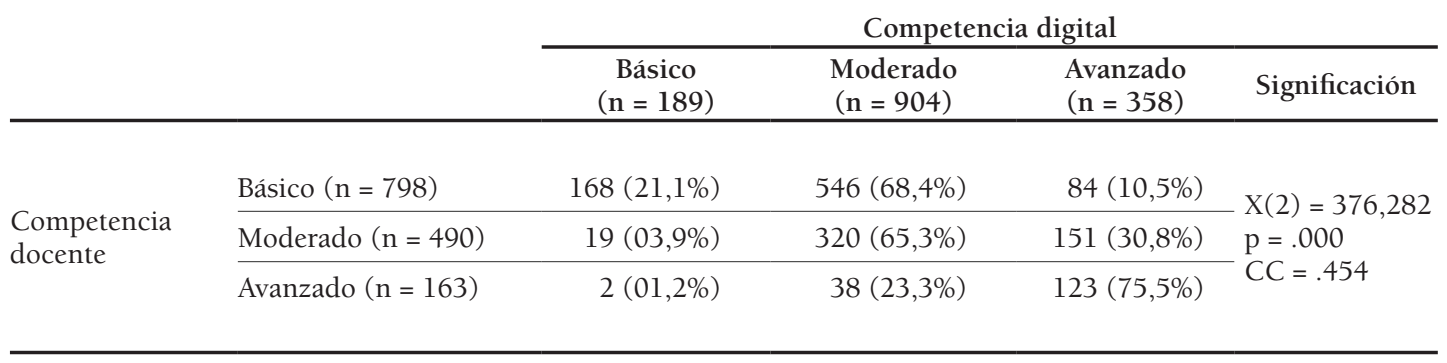


Tabla 4. Resultados de la partición en seis clústeres y ANOVA exploratorio Centros de conglomerados finales (6 grupos) ANOVA

\begin{tabular}{|c|c|c|c|c|c|c|c|c|c|c|c|c|c|c|}
\hline \multirow{2}{*}{$\begin{array}{l}\text { Factores de } \\
\text { preferencias de } \\
\text { formación }\end{array}$} & \multirow[b]{2}{*}{1} & \multirow[b]{2}{*}{2} & \multirow[b]{2}{*}{3} & \multirow[b]{2}{*}{4} & \multirow[b]{2}{*}{5} & \multicolumn{3}{|c|}{ Conglomerado } & \multicolumn{2}{|l|}{ Error } & & \multirow[b]{2}{*}{ Sig } & \multicolumn{2}{|c|}{ Tamaño del efecto } \\
\hline & & & & & & 6 & $\begin{array}{c}\text { Media } \\
\text { cuadrática }\end{array}$ & Gl & $\begin{array}{c}\text { Media } \\
\text { cuadrática }\end{array}$ & Gl & & & $\mathrm{d}_{\text {Cohen }}$ & $r$ \\
\hline Fl. Convencional & 7 & 6 & 2 & 2 & 4 & 5 & 1040.553 & 5 & 1.403 & 1619 & 741.404 & .000 & 4,2 & .902 \\
\hline F2. Autónoma & 7 & 4 & 2 & 6 & 6 & 3 & 845.648 & 5 & 1.503 & 1631 & 552.713 & .000 & 3,2 & .845 \\
\hline F3. Colaborativa & 7 & 3 & 2 & 2 & 5 & 6 & 949.990 & 5 & 1.463 & 1615 & 649.400 & .000 & 3,0 & .834 \\
\hline
\end{tabular}

Según estos resultados, el grupo 1 identifica al profesorado con una alta predisposición a todas las modalidades de formación en TIC, lo que nos lleva a considerarlos "todoterreno" (all-rounders). Este profesorado muestra una disposición a aprovechar cualquier modalidad de formación para aprender sobre TIC. Los grupos 5 y 6 muestran una predisposición al uso de modalidades colaborativas (basadas en la participación en proyectos, grupos y comunidades) para su formación en TIC de forma combinada con otra modalidad, haciéndolo el grupo 5 con estrategias más autodidactas y el grupo 6 con modalidades más convencionales. De otro lado, se encuentran los grupos 2 y 4 con un perfil muy definido hacia una modalidad formativa preferente, estando el grupo 2 altamente predispuesto a formarse bajo una modalidad convencional basada en la asistencia y participación en cursos, mientras que el grupo 4 muestra una mayor predisposición a formarse en TIC de forma autodidacta, indagando y experimentando con la propia tecnología. Por último, el grupo 3 revela un perfil de profesorado con una baja predisposición a formarse en TIC, sea cual sea la modalidad formativa, que hemos denominado "resistente". La tabla 5 muestra la distribución de estos perfiles en la muestra analizada.

La figura 3 ilustra las diferencias entre estos perfiles de profesorado en función de la competencia digital.

TABLA 5. Casos por conglomerado de profesorado según preferencia de formación en TIC

\begin{tabular}{|c|c|c|c|}
\hline \multirow{6}{*}{ Conglomerados } & 1. Todoterreno & 264 & $15,52 \%$ \\
\hline & 2. Convencional & 289 & $16,99 \%$ \\
\hline & 3. Resistente & 314 & $18,46 \%$ \\
\hline & 4. Autónomo & 211 & $12,40 \%$ \\
\hline & 5. Autónomo/colaborativo & 346 & $20,34 \%$ \\
\hline & 6. Colaborativo/convencional & 229 & $13,46 \%$ \\
\hline Casos válidos & & 1.653 & 97,185 \\
\hline Casos perdidos & & 48 & $2,82 \%$ \\
\hline Total & & 1.701 & $100,00 \%$ \\
\hline
\end{tabular}


Figura 3. Nivel de competencia digital en función de perfiles del profesorado en preferencias de aprendizaje en TIC

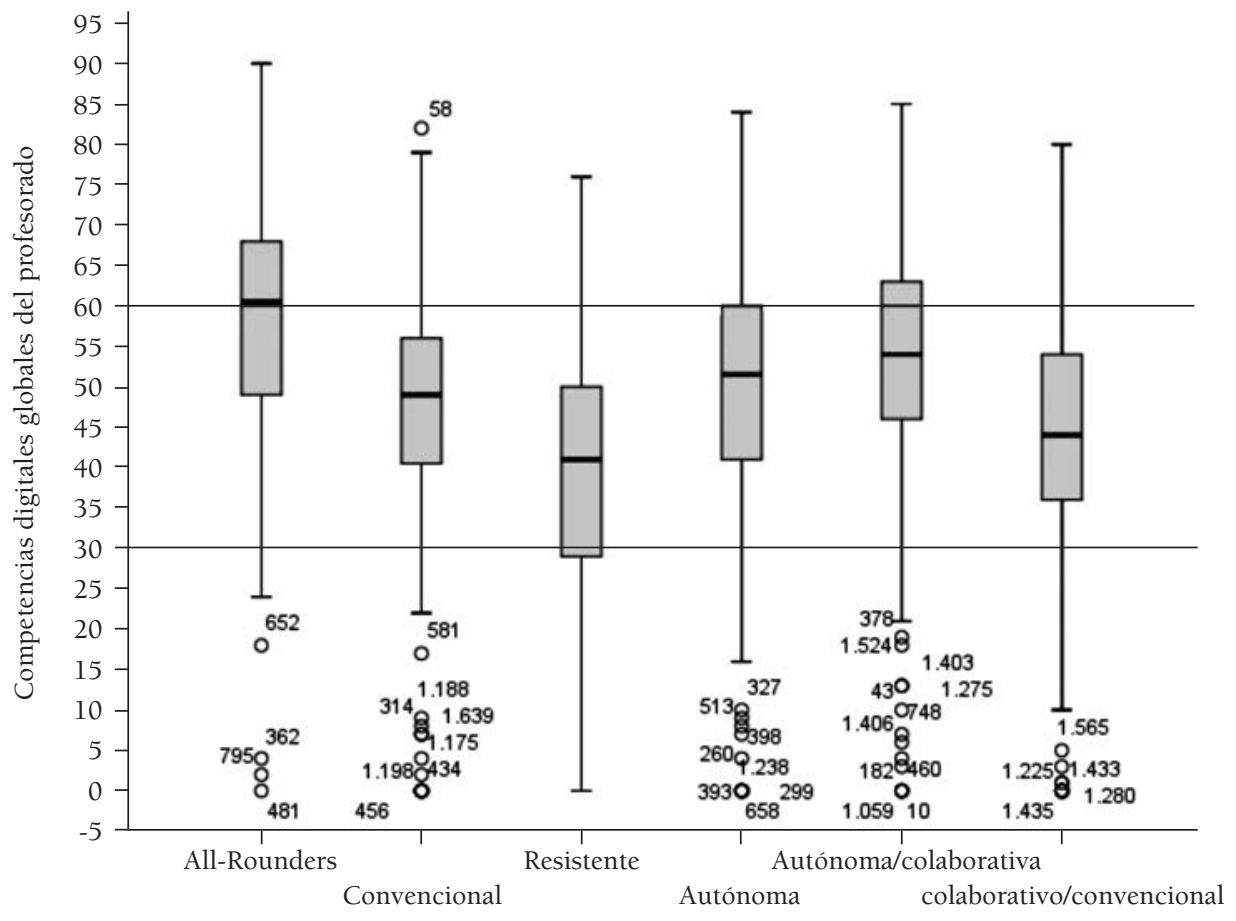

El profesorado todoterreno (all-rounders) es el que alcanza un nivel de competencia más elevado $(M=58,29 ; \mathrm{DT}=16,304)$, estando más del $50 \%$ de este grupo en un nivel avanzado de competencia digital. Le sigue el profesorado con preferencia a formarse de forma autónoma y colaborativa conjuntamente $(\mathrm{M}=53,07 ; \mathrm{DT}=$ 15,489) o solo de forma autónoma ( $M=49,20$; DT $=17,259)$, encontrando que el 35,7\% y $26,1 \%$, respectivamente, alcanzan un nivel avanzado de competencia digital.

En un nivel medio de competencia digital está el profesorado con preferencia a una formación convencional $(\mathrm{M}=47,16 ; \mathrm{DT}=15,571)$ o convencional-colaborativa $(\mathrm{M}=42,84$; $\mathrm{DT}=$ $17,258)$, donde el $70 \%$ del profesorado en estos perfiles alcanza un nivel moderado de competencia digital, siendo anecdótico el profesorado que alcanza un nivel avanzado.
Por último, el profesorado con una media en competencia digital más baja son los que muestran baja predisposición a formarse en TIC bajo cualquier modalidad $(\mathrm{M}=37,79 ; \mathrm{DT}=17,270)$, estando el $26,1 \%$ de este profesorado en un nivel básico de competencia digital.

Caracterización de perfiles de profesorado según nivel de competencia digital en las redes

Con el objeto de caracterizar estos perfiles de profesorado en función a la competencia digital, aplicamos análisis discriminante tomando como variable criterio los perfiles de profesorado en preferencias formativas y como variable predictora la competencia digital por dimensiones. Para ello, hemos considerado tres grupos: a) el profesorado todoterreno y 
autónomo-colaborativo, que alcanza una puntuación media-alta en competencia digital; b) el profesorado con preferencias a una modalidad convencional o colaborativa-convencional que alcanza una puntuación media de competencia digital; y c) el profesorado con baja predisposición a cualquier modalidad de formación en TIC (resistente) que alcanza una puntuación media-baja en competencia digital. Dos ecuaciones muestran bondad de ajuste con una adecuada correlación canónica para explicar las competencias digitales implicadas en la diferenciación de estos grupos.

De este modo, la ecuación discriminante para la diferenciación entre el profesorado todoterreno y el colaborativo-convencional muestra una adecuada correlación canónica de .356, con bondad de ajuste (Lambda de Wilks= $.873 ; \mathrm{p}=.000$ ), expresando que son las competencias tecnológicas y docentes las más determinantes en la diferenciación de estos grupos. La ecuación discriminante resultante se expresa en la siguiente fórmula: $\mathrm{y}=.262 \mathrm{tec}-$ nológica +.071 docente -2.803 . La ecuación excluye otras dimensiones predictoras por la multicolinealidad entre las mismas, asumiendo estas la función representativa del conjunto, como se puede observar en la matriz de estructura total (tabla 6).
Por su parte, la ecuación discriminante para la diferenciación entre el profesorado todoterreno y resistente también muestra una alta correlación canónica de .540 con una adecuada bondad de ajuste (Lambda de Wilks = .709; p = .000), expresando que son las competencias tecnológicas, socio-comunicativas y las de uso docente las más determinantes en la diferenciación de estos grupos. La ecuación discriminante resultante se expresa en la siguiente fórmula: $\mathrm{y}=.188$ CD tecnológica +.077 CD sociocomunicativa $+.074 \mathrm{CD}$ docente -2.525 , aunque, como hemos señalado anteriormente, la multicolinealidad entre las variables predictoras puede haber excluido otras de la ecuación discriminante (tabla 6).

Aplicado el Anova de Kruskal-Wallis, encontramos que las diferencias observadas entre los perfiles formativos de profesorado en el nivel de competencias son estadísticamente significativas $(X 2=226,233 ; p=.000)$. Para valorar el tamaño del efecto de las preferencias formativas sobre las competencias digitales, comparamos los grupos más extremos (all-rounders vs. resistentes), obteniendo una significativa diferencia entre ambos (U de Mann-Whitney = 10852,500; $\mathrm{p}=.000)$. El tamaño del efecto es bastante elevado, como muestran los coeficientes calculados $(\mathrm{d}$ de Cohen $=1,2 ; \mathrm{r}=.521)$.

TABla 6. Matriz de estructura total de las funciones discriminantes

\begin{tabular}{|c|c|c|c|}
\hline \multicolumn{2}{|c|}{$\begin{array}{l}\text { Matriz de estructura total discriminante } 1 \\
\text { Todoterreno con colaborativo-convencional }\end{array}$} & \multicolumn{2}{|c|}{$\begin{array}{l}\text { Matriz de estructura total discriminante } 2 \\
\text { Todoterreno con resistente }\end{array}$} \\
\hline \multirow{2}{*}{ Matriz de estructura total } & Función & \multirow{2}{*}{ Matriz de estructura total } & Función \\
\hline & 1 & & 1 \\
\hline Competencia tecnológica & ,965 & Competencia tecnológica & ,938 \\
\hline Competencia cognitiva &, 726 & Competencia sociocomunicativa & 850 \\
\hline Competencia sociocomunicativa &, 717 & Competencia cognitiva &, 774 \\
\hline Competencia ética &, 570 & Competencia emocional & 659 \\
\hline Competencia emocional &, 551 & Competencia ética & ,626 \\
\hline Competencia docente &, 551 & Competencia docente & ,586 \\
\hline
\end{tabular}




\section{Discusión y conclusiones}

Los resultados de este estudio muestran que el profesorado en activo alcanza un nivel moderado de competencia digital en el uso de las redes sociales, siendo las competencias docentes las que tiene menos desarrolladas. Esto concuerda con los resultados de estudios previos (Almerich et al., 2011a; Suárez et al., 2013) en los que se evidencia un mayor nivel en competencias tecnológicas que docentes a nivel de aula. Nuestros resultados indican que un muy pequeño porcentaje de profesorado hace uso de las redes sociales en su actividad docente, lo que es coincidente con lo demostrado por Cortina-Pérez et al. (2014) o San Nicolás et al. (2012) que documentan el escaso uso docente que hace el profesorado de las herramientas de la Web 2.0. No hemos observado diferencias en el nivel de competencias digitales de uso de las redes sociales en función del género pero sí en función de la edad. Estos resultados concuerdan solo parcialmente con los de estudios previos (Suárez et al., 2013; Almerich et al., 2011a) en los que se ha señalado el género y la edad como factores que inciden en las competencias tecnológicas del profesorado, encontrando que es el profesorado más joven el que mayor nivel de competencia alcanza. Esta diferencia puede deberse al hecho de que nuestro estudio se haya centrado exclusivamente en las competencias de uso de las redes sociales y no en las TIC en general. Algunas investigaciones internacionales (Clipson et al., 2012; Mazman y Uzluel, 2011) han documentado cómo la brecha digital de género se reduce en el uso de las redes sociales virtuales donde las mujeres tienen mayor protagonismo. Por otra parte, también hemos podido constatar que la mayor parte del profesorado con puntuaciones altas en competencia digital usa las redes en su docencia, por lo que parece importante considerar como objetivo de la formación del profesorado el logro de este nivel avanzado de competencia digital.

Por otra parte, con nuestro estudio demostramos la existencia de relación entre preferencias de formación en TIC y la competencia digital de uso de las redes sociales, siendo el profesorado con predisposición hacia una heterogeneidad de modalidades formativas el que alcanza un nivel más elevado de competencia digital seguido del que se muestra predispuesto a hacerlo bajo estrategias colaborativas y autodidactas conjuntamente. Este resultado viene a respaldar las políticas en materia de formación del profesorado que apuestan por diversificar la oferta formativa en cuanto a metodologías. Este resultado contrasta con los de algunos estudios previos (Wastiau et al., 2013) que sugerían que el profesorado prefería métodos informales y de base práctica para su formación. Nuestros resultados matizan este conocimiento previo, al mostrar una diversidad de perfiles de profesorado en función de sus preferencias de formación que tienen impacto en su nivel de competencia digital, lo que nos permite concluir que el profesorado aprende sobre TIC de muchas maneras y que es precisamente aquel que lo hace así el que alcanza mejores puntuaciones en competencia digital. También se puede extraer de los resultados de nuestro estudio que el profesorado con una marcada preferencia hacia una formación convencional, colaborativa o autónoma exclusivamente alcanza un nivel medio de competencia digital que afecta a sus posibilidades reales de aplicación docente en el aula.

Los resultados también muestran que la competencia tecnológica y la docente son directamente responsables del logro de un nivel avanzado de competencia digital, lo que conduce a plantear que la formación contemple ambas dimensiones. Estudios previos (Suárez et al., 2013) han demostrado que las competencias tecnológicas inciden en las pedagógicas aumentando la confianza del profesorado en sus habilidades y que es este el que más actividades con TIC emprende con su alumnado. Por ello, diversos trabajos (Almerich et al., 2011b; Cabero, 2014) insisten en la importancia de centrar la formación en las aplicaciones docentes de las TIC sin olvidar la formación tecnológica. 


\section{Referencias bibliográficas}

Almerich, G., Suárez-Rodríguez, J. M., Jornet, J. M., y Orellana, N. (2011a). Las competencias y el uso de las Tecnologías de la Información y Comunicación por el profesorado: estructura dimensional. Revista Electrónica de Investigación Educativa, 13 (1), 28-42. Recuperado de http://redie. uabc.mx/index.php/redie/article/view/269/709

Almerich, G. Suárez-Rodríguez, J. M., Belloch, C., y Bo, R. M. (2011b). Las necesidades formativas del profesorado en TIC: perfiles formativos y elementos de complejidad. RELIEVE, 17 (2), 1-28. Recuperado de http://www.uv.es/relieve/v17n2/RELIEVEv17n2_1.htm

Area, M., y Pessoa, T. (2012). De lo sólido a lo líquido: Las nuevas alfabetizaciones ante los cambios culturales de la Web 2.0. Comunicar, 38, 13-20. doi:10.3916/C38-2012-02-01

Cabero, J. (2014). Formación del profesorado universitario en TIC. Aplicación del método Delphi para la selección de los contenidos formativos. Educación XXI, 17 (1), 111-132. doi: 10.5944/ educxx1.17.1.10707

Castañeda, L., y Adell, J. (2013). Entornos personales de aprendizaje: claves para el ecosistema educativo en red. Madrid: Alcoy.

Clipson, T. W., Wilson, S. A., y Dufrene, D. D. (2012). The Social Networking Arena: Battle of the Sexes. Business Communication Quarterly, 75 (1), 64-67. doi: 10.117-7/1080569911423961

Coll, C., y Engel, A. (2014). Introduction: Personal Learning Environments in the context of formal education / Introducción: los Entornos Personales de Aprendizaje en contextos de educación formal. Cultura y Educación: Culture and Education, 26 (4), 617-630. doi: 10.1080/11356405.2014.985947

Comisión Europea (2015). Education and training monitor 2014. Luxembourg: Publications Office of the European Union. Recuperado de: http://ec.europa.eu/education/library/publications/ monitor15_en.pdf

Cortina-Pérez, B., Gallardo-Vigil, M. A., Jiménez-Jiménez, M. A., y Trujillo-Torres, J. M. (2014). Digital illiteracy: a challenge for 21st century teachers. Cultura y Educación, 26 (2), 231-264. doi: $10.1080 / 11356405.2014 .935108$

DiMaggio, P., Hargittai, E., Celeste, C., y Shafer, S. (2004). Digital inequality: From unequal access to differentiated use. En K. Neckerman (ed.), Social Inequality (pp. 355-400). New York: Russell Sage Foundation.

Fahser-Herro, D., y Steinkuehler, C. (2010). Web 2.0 Literacy and Secondary Teacher Education. Journal of Computing in Teacher Education, 26 (2), 55-62.

Ferrari, A. (2013). DIGCOMP a framework for developing and understanding digital competence in Europe. (Y. Punie y B.N. Brecko, Eds.). Luxembourg: Publications Office. Recuperado de http:// ipts.jrc.ec.europa.eu/publications/pub.cfm?id=6359

Greenhow, C., y Robelia, B. (2009). Informal learning and identity formation in online social networks. Learning, Media and Technology, 34 (2), 119-140. doi: 10.1080/17439880902923580

Gruszczynska, A., Merchant, G., y Pountney, R. (2013). Digital Futures in Teacher Education. Exploring open approaches towards digital literacy. Electronic Journal of e-Learning, 11 (3), 193-206.

Hargittai, E., y Hinnant, A. (2008). Digital Inequality: Differences in Young Adults' Use of the Internet. Communication Research, 35, 602-621.

Howard, M. C. (2014). Creation of a computer self-efficacy measure: analysis of internal consistency, psychometric properties, and validity. Cyberpsychology, behavior, and social networking, 17 (10), 677-681. doi: 10.1089/cyber.2014.0255

Hughes, J. E., Ko, Y., Lim, M., y Liu, S. (2015). Preservice Teachers' Social Networking Use, Concerns, and Educational Possibilities: Trends from 2008-2012. Journal of Technology and Teacher Education, 23 (2), 185-212. Recuperado de http://www.editlib.org/p/130448 
Relación entre las preferencias de formación del profesorado y su competencia digital en las redes sociales

INTEF (2013). Marco común de competencia digital docente. Madrid: MECD. Recuperado de: http://educalab.es/documents/10180/12809/MarcoComunCompeDigiDoceV2.pdf

Janssen, J., Stoyanov, S., Ferrari, A., Punie, Y., Pannekeet, K. y Sloep, P. (2013). Experts' views on digital competence: Commonalities and differences. Computers \& Education, 68, 473-481. doi:10.1016/j.compedu.2013.06.008

Keith, H. (2013). Using Facebook and other SNSs in K12 classrooms: ethical considerations for safe social networking. Issues in Teacher Education, 22 (2), 39-54.

Livingstone, S., y Helsper, E. (2007). Gradations in digital inclusion: children, young people and the digital divide. New Media y Society, 9 (4), 671- 696. doi: 10.1177/1461444807080335

Mazman, S. G., y Usluel, Y. K. (2011). Gender Differences in Using Social Networks. TOJET: The Turkish Online Journal of Educational Technology, 10 (2), 133-139. Recuperado de http://goo.gl/8o42eT

OCDE (2014). TALIS 2013 Results: An international perspective on teaching and learning, TALIS, OCDE Publishing. doi:10.1787/9789264196261-en

Rebollo-Catalán, Vico-Bosch y García-Pérez, R. (2015). El aprendizaje de las mujeres de las redes sociales y su incidencia en la competencia digital. Prisma social: Revista de Ciencias Sociales, 15, 122-146.

Sadaf, A., Newby, T. J. y Ermer, P. A. (2013). Exploring factors that predict preservice teachers' intentions to use Web 2.0 technologies using decomposed theory of planned behavior. Journal of Research on Technology in Education, 45 (2), 171-196.doi:10.1080/15391523.2012.10782602

San Nicolás, M. B., Fariña, E., y Area, M. (2012). Competencias digitales del profesorado y alumnado en el desarrollo de la docencia virtual. Revista Historia de la Educación Latinoamericana, 14 (19), 227-245. Recuperado de http://revistas.uptc.edu.co/revistas/index.php/historia_educacion_latinamerican/article/view/1993/1988

Suárez Rodríguez, J. M., Almerich, G., Gargallo, B., y Aliaga, F. M. (2013). Las competencias del profesorado en TIC: estructura básica. Educación XXI, 16 (1), 39-62. doi:10.5944/educXX1.16.1.716

Tyner, K., Gutiérrez-Martín, A., y Torrego-González, A. (2015). "Multialfabetización" sin muros en la era de la convergencia. La competencia digital y la cultura del hacer como un revulsivo para una educación continua. Profesorado, 19 (2), 42-56.

Ugur, K. (2014). Can they plan to teach with Web 2.0? Future Teachers' potential use of the emerging Web. Technology, Pedagogy and Education, 23 (4), 471-489. doi:10.1080/1475939X.2013.813408

Vergés, N., Hache, A., y Cruells, E. (2011). Indagando en la relevancia de Internet en el acceso, uso y deseos de las TIC por parte de las mujeres en las TIC. Teoría de la Educación: Educación y Cultura en la Sociedad de la Información, 12 (2), 105-121.

Wang, D., Xu, L., y Chan, H. (2015). Understanding the continuance use of social network sites: a computer self-efficacy perspective. Behavioury Information Technology, 34 (2), 204-216. doi: 10.1080/0144929X.2014.952778

Wastiau, P., Blamire, R., Kearney, C., Quittre, V., Van de Gaer, E., y Monseur, C. (2013). The Use of ICT in Education: a survey of schools in Europe. European Journal of Education, 48 (1), 11-27. doi: 10.1111/ejed.12020

\section{Abstract}

The relationship between teacher training preferences and their digital skills on social networks

INTRODUCTION. Several international reports have revealed the importance of teacher education in ICT as a key factor to innovation and educational improvement. Recent studies analyze the teachers' skills to take advantage the educational potential of these tools. In this 
paper, we try to study the relation between teachers' training preferences and their digital skills in social networks, allowing us to discover which of the strategies for teacher education are the most effective to achieve an advanced level of digital competence. METHOD. We have carried out a research based on a survey to 1701 teachers from 114 schools in the area CEP AlgecirasLa Línea, Cádiz (Andalucía, España). Two scales were applied to measure digital competences and learning preferences, which got reliability coefficients of .92 and .82 respectively. RESULTS. Teachers show a moderate level of digital skills, of which teaching skills are the less developed. Teachers with a good attitude towards the combined use of all training strategies and teachers, who prefer collaborative learning strategies and independent learning jointly, are those that express an advanced level of digital competence. We didn't observe differences in digital competence of teachers depending on gender but we found them depending on age. DISCUSSION. These results are consistent with other research on teacher education in ICT, which show deficiencies in teacher education in order to do a better pedagogical use of ICT. This research provides some evidences which allow us to extract conclusions to guide teacher training in ICT.

Keywords: Teacher competencies, Technology uses in education, Social networks, Web 2.0 technologies, Teacher education, Digital competence.

\section{Résumé}

La relation entre les préferences de formation des enseignants et leurs compéténces numériques sur les reseaux sociaux

INTRODUCTION. Plusieurs informes ont montré l'importance de la formation des enseignants aux TIC comme un facteur clé pour l'innovation et l'amélioration de la pratique éducative. Études récents analysent les compétences des enseignants qui permettent une meilleure exploitation pédagogique des technologies numériques. Dans cet article on analyse la relation entre les préférences de formation des enseignants et leurs compétences numériques dans l'utilisation des réseaux sociaux, ce qui nous permet découvrir quelles sont les modalités de formation les plus efficaces pour obtenir un niveau avancé de compétence numérique. MÉTHODE. La recherche est basée sur une enquête constitué para un échantillon pondéré à partir d'une population de 1701 enseignants procédant de 114 centres éducatifs de la zone CEP Algésiras-La Línea, Cadiz (Andalousie, Espagne). On a utilisé deux échelles: une de compétences numériques et une autre de préférences d'apprentissage dessinés ad hoc avec coefficient de fiabilité de .92 et .82 . RÉSULTATS. Les enseignants montrent un niveau moyen de compétences numériques et parmi elles las compétences d'y enseignement son las moins développées. Les enseignants qui ont une haute prédisposition aux nombreuses modalités de formation et ceux qui préfèrent les modalités collaboratives d'enseignement mêlés au apprentissage autonome sont les enseignants qui ont un niveau plus haute de compétence numérique. On n'a pas obtenu de différences dans la compétence numérique des enseignants par rapport au genre mais par rapport à l'âge. DISCUSSION. Ces résultats sont conformes à ceux qui proviennent d'autres recherches sur la formation des enseignants aux TIC et qui montrent que la formation des enseignants a encore de faiblesses qui empêchent une meilleure exploitation pédagogique des TIC.

Mots clés: Compétences des enseignants, Utilisation des technologies dans l'éducation, Réseaux sociaux, Technologies web 2.0, Formation des enseignants, Compétence numérique. 
Relación entre las preferencias de formación del profesorado y su competencia digital en las redes sociales

\section{Perfil profesional de los autores}

\section{Rafael García-Pérez (autor de contacto)}

Profesor titular de la Universidad de Sevilla, especialista en Métodos de Investigación y Diagnóstico en Educación. Su línea de investigación se centra en técnicas e instrumentos de diagnóstico sobre género, tecnología y educación. Ha desarrollado proyectos de innovación con TIC en colaboración con los Centros de Profesorado.

Correo electrónico de contacto: rafaelgarcia@us.es

Dirección para la correspondencia: Dpto. Métodos de Investigación y Diagnóstico en Educación. Facultad de Ciencias de la Educación, Universidad de Sevilla, C/ Pirotecnia, s/n, CP 41013, Sevilla.

\section{Ángeles Rebollo-Catalán}

Profesora titular de la Universidad de Sevilla en Métodos de Investigación y Diagnóstico en Educación. Su línea de trabajo se vincula al estudio de los procesos de innovación educativa mediados por las tecnologías y el género. Su actividad científica más reciente se vincula a dos proyectos de I+D y un proyecto financiado por la Fundación BBVA vinculados a la inclusión digital y los procesos de aprendizaje en las redes sociales.

Correo electrónico de contacto: rebollo@us.es

\section{Curro García-Pérez}

Director del CEP Algeciras-La Línea desde 2009, participando en este centro, desde 2005, como asesor de secundaria para la formación del profesorado y vicedirector (2006-2009). Profesor de enseñanza secundaria desde 1989, cuenta con una larga trayectoria directiva habiendo sido secretario, jefe de estudios, vicedirector y director en el IES Mar de Poniente-La Línea de la Concepción.

Correo electrónico de contacto: curro.garciaperez@uca.es 